\title{
A CROSS-SECTIONAL SURVEY ON PREVALENCE OF UPPER CROSS SYNDROME AND ITS CORRELATION TO WRMSDS IN WORKING PHYSIOTHERAPISTS
}

\begin{abstract}
\section{BACKGROUND AND AIMS}

Upper Cross Syndrome (UCS) is stress over the neck region due to poor posture and ergonomics, particularly sitting or standing. This study aimed to determine the prevalence of UCS in working physiotherapists of Pakistan and its correlation of UCS with WRMSDs to gauge the burden of the Musculoskeletal disorders (MSDs).

\section{METHODOLOGY}

A cross-sectional study was conducted on working physiotherapists recruited through convenience sampling technique via email or social media platforms. A selfadministered questionnaire constitutes of screening questions regarding posture and Modified Oswestry Neck Disability Index was used to collect data.

\section{RESULTS}

A total number of 148 physiotherapists participated in the study out of which 45 males and 103 females. The professionals were categorized on basis of their level of experience years $40 \%$ beginners $32 \%$ competent and $28 \%$ proficient. The screening revealed that $75(51.7 \%)$ have forwarded head posture while 73 (49.3\%) physiotherapists suffered from moderate disability. The chi-square analysis was found to have significant association $(p<0.005)$ on gender, experience level and duration of working posture hour with disability.

\section{CONCLUSION}

It was concluded that UCS prevalence was found to be $27 \%$ in working physiotherapists whereas female and individuals working for prolonged duration were found to be more prone to develop UCS. Further studies should be conducted taking large sample size and confounding factors for the better understanding of the health-related outcomes.

\section{KEYWORDS}

Upper Cross Syndrome, Forward Head Posture, Rounded Shoulder, Neck Pain, Work Related Musculoskeletal Disorders, Upper Trapezius.
Mariyam Mubashir

Physiotherapist

Freelancer

mariyamkaanch@hotmail.com
[Mubashir M, A Cross-Sectional Survey on Prevalence of Upper Cross Syndrome and Its Correlation To WRMSDs in Working Physiotherapists .Pak.j.rehabil.2021;10(1);-5159]

DOI; 10.36283/pjr.zu.10.1/008 


\section{INTRODUCTION}

According to the World Health Organization, Musculoskeletal Disorders are one of the major factors that lead to disability among people of age 20-50 years, that are mostly related to their work which are headaches and chronic neck pain the most common complaint around the globe ${ }^{1,2}$. The disability is recognized to cause serious medical and socioeconomic ailments, estimated the loss as high as $2 \%$ of Gross Domestic Product (GDP) due to absenteeism and low productivity ${ }^{3,4}$. Number of studies revealed that approximately $90 \%$ of rehabilitation professionals have musculoskeletal ailments among which $50 \%$ physiotherapists tends to suffer from Work-Related Musculoskeletal Disorders (WMSDs) during 5 years practice ${ }^{5,6}$. This may be due to their nature of work that includes prolonged standing/sitting/lifting or performing manual techniques in clinical or academic setting ${ }^{5,6}$ that constitutes the high prevalence $(66 \%)$ in lower limb and especially low back and (61\%) neck region.

Cervical Cross Syndrome, referred to as Upper Cross Syndrome (UCS) is described as a predictive pattern of alternative tightness and weakness of Upper Trapezius, Pectoralis Major, Levator Scapulae, Rhomboids, Serratus Anterior and Deep Flexors of the neck, particularly Scalene muscles caused due to poor working habits, inappropriate body ergonomics, or low self-esteem ${ }^{7,8,9}$. Upper Cross Syndrome is a characterized by muscular imbalance of skeletal muscles activation and inhibition rather than just single muscle involvement. Any failure of activation between tonic and phasic muscles results in muscular insufficiencies ${ }^{10}$. Consequently, neck and upper back pain is the foremost complaint of UCS, mostly diagnosed as Cervicogenic Vertigo with shoulder pathology in number of patients ${ }^{11}$. Studies have revealed that prevalence of Work Related
Musculoskeletal Disorders (WRMSDs) is higher among physiotherapists i.e. 54.8\% of suffered from the condition in a private working environment ${ }^{12}$. This may be due to the strenuous activities in relation to a profession that has found to be a predominant risk factor for Musculoskeletal Disorders MSDs. A study conducted by Rahimi et al $^{13}$ found that 94\% Iranian physiotherapists suffered from Work Related Musculoskeletal Disorders (WRMSDs) that enlightened lumbar pain $(65 \%)$, neck pain $(57.4 \%)$, shoulder (50.2\%), upper back (49\%), and knee $(45.5 \%)$ respectively due to routine activities that leads physiotherapists at a risk of Upper Cross Syndrome (UCS) ${ }^{14,15,16}$. Despite of the fact, Physiotherapist (PTs) are alleged to have adequate knowledge of Musculoskeletal Disorders (MSDs) yet are at the high risk of developing WMSDs. To date, there are many studies reported to ascertain the prevalence of WorkRelated Musculoskeletal Disorders (WRMDs) among rehab professionals, however there is scarcity of data on the prevalence of Upper Cross Syndrome (UCS) among physiotherapists in Pakistan. Hence, this study is aimed to determine the prevalence of Upper Cross Syndrome (UCS) in working physiotherapists of Pakistan and to evaluate the correlation of UCS with WRMSDs to gauge the burden of the MSDs in order to strategize an effective prevention and control approaches.

\section{METHODOLOGY}

\section{Study Setting}

This study was conducted at Rehabilitation Department of primary and tertiary care hospitals of Pakistan including rehabilitation institutes.

\section{Target Population}

Working Physiotherapists with minimum experience of two years or beginner level of experience.

\section{Study Design}

Cross-sectional study. 


\section{Duration of Study}

6 to 8 months.

\section{Sampling Technique}

Non-Probability Convenience Sampling Technique

\section{Sample Size}

Sample size is calculated by using Open EPI software. Therefore, at anticipated frequency of $50 \%$ with confidence interval of $95 \%$ with margin of error at $5 \%$, a sample size of $n=148$ was calculated by using formula.

\section{Sample Selection}

\section{Inclusion criteria}

- $\quad$ Both male and female working physiotherapists from beginner to proficient level of experience between 24 to 50 years.

\section{Exclusion criteria}

- Novice level of experience

- Recent trauma, surgery, fractures to related joints, soft tissue malignancy, and congenital deformities.

\section{Data Collection Tool}

Data was collected through Modified Oswestry Neck Disability Index (MONDI), a useful standard tool in the assessment of neck pain disability designed assess neck pain and functional limitations related to it. The responses on index is comprised on a 6-point Likert scale from 0-5 ranging from 'never' to 'worst imaginable' on the questions of ten sections, each with a possible score of 5 . The final score was calculated on the responses in the questionnaire expressed in percentage as follows;

0-4 Points (0-8\%) No Disability.

5-14 Points (10 - 28\%) Mild Disability.

15-24 Points (30-48\%) Moderate Disability.

25-34 Points (50-64\%) Severe Disability.

35-50 Points (70-100\%) Complete Disability.

\section{Data Collection Procedure}

Data was collected from physiotherapists working in primary and tertiary care settings of Pakistan as per the inclusion criteria. Consent was taken from them prior to data collection to ensure their voluntary consent. Afterwards, link to Google form questionnaire was sent to them using email and WhatsApp and on various physiotherapy social media (Facebook, Instagram, LinkedIn) platforms. Moreover, participants were reminded to fill the questionnaire followed by soft reminders. The questionnaire comprises of demographic details, qualification, designation, years of experience or year of training, hours of work per week, experiencing any neck or shoulder discomfort and do they have drooping shoulders or poking chin followed by assessment of neck disability index on 6-point Likert scale from 0-5 (Never, mild, moderate, fairly severe, very severe, worst imaginable). Modified Oswestry Neck Disability Index (MONDI) is a standard tool designed to determine neck pain and functional restrictions related to it. Responses from the participants were analyzed thereafter

\section{Data Analysis Strategy}

Data were entered and analyzed using SPSS (Statistical Package for Social Sciences) version 20. The demographic characteristics of the participants are represented through frequency, mean and standard deviations whereas the participant responses will be evaluated through frequency and percentage. The cross-tab and chi-square statistics was applied to determine the association as per the described objectives.

\section{Ethical Considerations}

The researcher provided complete information about the study to each and every participant before start of the study. The information procured from the participants was kept as confidential information. Every participant has full right of withdrawing the study at any time during the study. This study has no harm 
to candidate and consent was taken prior to study.

\section{RESULTS}

A total number of $n=148$ physiotherapists participated in the study, included 45 males and 103 females with highest age range reported in $20-30$ years (62.8\%), 0-2 years' experience (37.8\%) and 40-60 hours/week (40.5\%) as described in Table1, Figure-1.

\section{Table-1 Demographic Characteristics ( $n=148)$

Experience Level

\begin{tabular}{cc} 
Beginner & $93(62.8 \%)$ \\
\hline \hline Competent & $50(33.7 \%)$ \\
\hline \hline Proficient & $5(3.5 \%)$ \\
\hline Working Hours/Week & $33(22.3 \%)$ \\
\hline \hline $0-20$ & \\
\hline hours/week & $48(32.4 \%)$ \\
\hline \hline $\begin{array}{c}30-40 \\
\text { hours/week }\end{array}$ & $60(40.5 \%)$ \\
\hline \hline $40-60$ & $7(4.7 \%)$ \\
\hline hours/week & \\
\hline \hline & \\
\hline \hline & 60 \\
hours/week & \\
\hline \hline
\end{tabular}

The screening revealed that 94 (63.5\%) have palm face inwards towards the body whereas 75(50.7 \%) have forwarded head posture as shown in Figure 1 and 2. Moreover, the assessment of disability interpretation On MONDI revealed that 97 (65.5\%) physiotherapists suffered from moderate disability, 40 (27\%) severe, 7 (4.7\%) crippled, 3 (2\%) minimum while only $1(0.75)$ has exaggerated symptoms as shown in Figure-3.
Figure-1 represents the posture analysis according to participant's perception

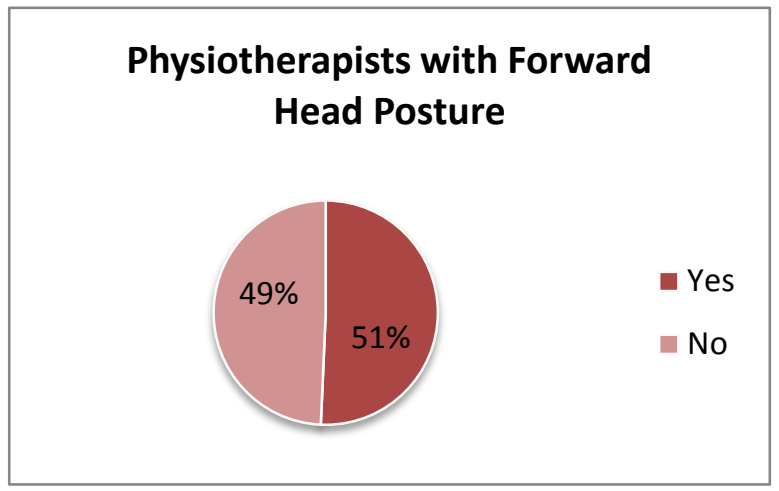

Figure- 2 represents the screening of forward head posture in participants
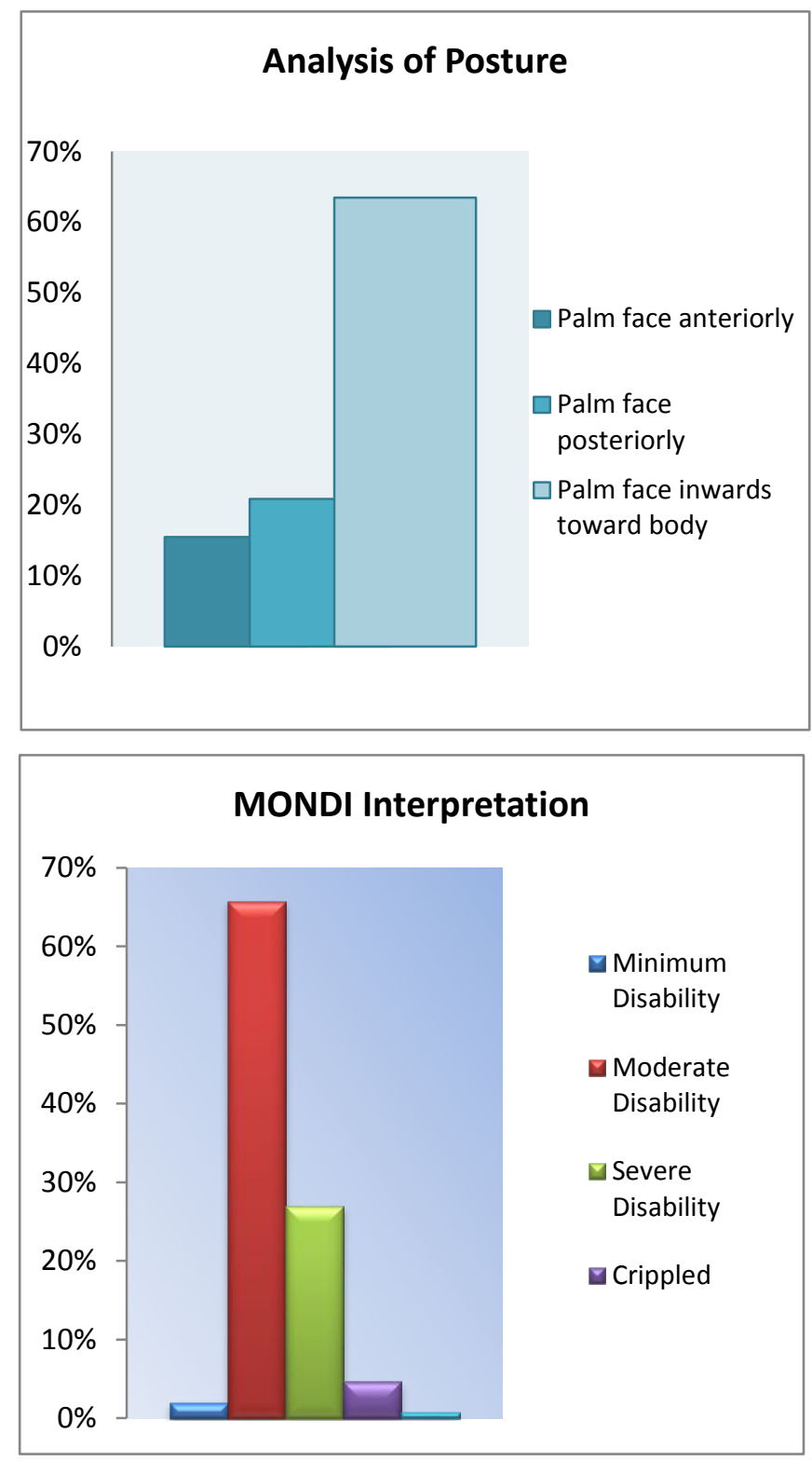

Figure-3 shows severity of disability on Modified Oswestry Neck Disability Index 
The cross-tab analysis revealed that among 20-30 years individuals, 64 has moderate disability and 26 has severe disability that determines the significant association $(p<0.005)$ on chi-square statistics on age whereas 68 females reported to have moderate and 32 has severe disability respectively as compared to male amongst which only 29 has moderate, 8 has severe and 2 has minimum disability. It was further revealed that individuals who worked for 40-60 hours/week have moderate disability, followed $30-40$ and $0-20$ hours/week determines non-significant association while 40 to 28 individuals with forward head posture reported to have moderate to severe disability. Thus, found to have significant association $(p<0.005)$.

\section{Table-2 Chi-Square Test of}

Association

\begin{tabular}{cccc}
\hline $\begin{array}{c}\text { Variable } \\
\text { s }\end{array}$ & $\begin{array}{c}\text { Pearson } \\
\text { Chi-square }\end{array}$ & Df & Sig. \\
$\begin{array}{c}\text { Gender } \\
\text { Disabilit } \\
\text { y }\end{array}$ & 11.779 & 4 & $* 0.019$ \\
$\begin{array}{c}\text { Age-Disability } \\
\begin{array}{c}\text { Forward Head } \\
\text { Posture-Disability }\end{array}\end{array}$ & 28.547 & 12 & $* 0.005$ \\
$\begin{array}{c}\text { Working } \\
\text { Hours/Week- } \\
\text { Disability }\end{array}$ & 9.055 & 12 & 0.698 \\
\hline
\end{tabular}

$*_{p}<0.05$ significant association

Therefore, the total prevalence of UCS in physiotherapists was estimated to be $27 \%$, the individuals reported to have moderate disability with respect to gender, working hours and poor posture.

\section{DISCUSSION}

The findings of this study revealed the prevalence of $27 \%$ of Upper Cross syndrome (UCS) in physiotherapists of Pakistan. It was revealed that female gender was more vulnerable to develop the disorder while prolonged working hours showed significant association to disability the reason behind this might be the poor posture and weak musculature. Similarly, a study conducted by Trinkoff et al ${ }^{17}$ determined the relationship between prolonged working duration and musculoskeletal problems among registered nurses showed that $13+$ hours per day, off shifts, weekend work, overtime or continuous working are the factors to develop musculoskeletal disorders along this low salaries and psychological stress increase the burden of Work-Related Musculoskeletal Disorders (WRMDs). Therefore, reduced hours and relax shifts are required to minimize the risk of Musculoskeletal Disorders (MSDs) and recovery time. Likewise, our study reported that most of the individuals worked for 40-60 hours/week have moderate disability therefore certain measures has be to taken for the lifelong consequences. Another study conducted by Tolu and Basaran 18 declared that $98.4 \%$ healthcare professionals who worked for longer duration in operation theater or emergencies reported to have complaints of muscular deficiency developed within 1 year, in particular with low back disorders (70.7\%). Furthermore, it was revealed that women were more likely to develop Work Related Musculoskeletal Disorders (WRMDs) in the upper back this might be due to weak upper limb posture than in men, similar to this study as women were found to be prone to Upper Cross Syndrome (UCS). Therefore, inappropriate postural position may be serving as predominant risk factor in Musculoskeletal Disorders (MSDs) development. 
A study conducted by Daneshmandi ${ }^{19}$ determined the association between Upper Cross syndrome (UCS) and trained body builders. The analysis revealed the measurements of forward head inclination, rounded shoulder and upper back kyphosis was found to be increased in trained body builders then the untrained. The concept was in contrast with the general assumption, that body building is a sport for growth and development of a symmetrical muscle form rather it may cause imbalance between tonic and phasic muscles of the body. Moreover, lack of proper stretching before and after training can also results in reduced shoulder mobility, increasing muscle bulk can also reduce the mobility of limbs. Thus, Upper Cross syndrome (UCS) may also be seen prevalent among such professions yet it is unexplored till date. Besides, Shahid et $\mathrm{al}^{20}$ also determined the association forward head posture on neck disability and associated level of stress among undergraduate students in which 70 (56\%) students presented with forward chin posture, $3(4.2 \%)$ students had moderate disability, $37(52.8 \%)$ students with severe disability were reported. Whereas, 23 (32.8\% had low, 30 (42.8\%) moderate and 17 (24.2\%) high stress. There was a significant co relation with forward head posture and level of stress but no effect on functional disability. Although, similar to these findings, our study reported more than $50 \%$ individuals with forward neck posture and moderate disability, with significant association.

Furthermore, number of studies reported that physiotherapists have frequent workrelated injuries that are often unreported due to busy schedule or to avoid job incompetence. Moreover, the most affected body regions are lower back, upper back, shoulders and neck respectively. It was also revealed that more than $89 \%$ therapists working in private settings were most affected with
Work Related Musculoskeletal Disorders (WRMDs) within 5 years of working but pushed to continue working while they had Musculoskeletal Disorders (MSDs) was major risk factors in addition with slow economic status and psychological issues $^{21}$. However, no such analysis has been done in our study to report that which sector of physiotherapists are more prone to develop Musculoskeletal Disorders (MSDs) with respect to age, gender, working hour, posture, psychological issues and work performance respectively.

Prevalence of Upper Cross syndrome (UCS) has been studied among students as well, a study that concluded that medical students who did not give attention to their postures, prolong duration of classes and long term time spending on laptops/ personal computer led them to develop Upper Cross syndrome (UCS) in their future, was found to be $37.1 \% 22$. This condition is not only prevalent among professional health care workers but also related in abundance with the labors in various sectors of the industry. Mujawar, and Sagar23 have figured out a prevalence of Upper Cross syndrome (UCS) being $28 \%$ in laundry workers owing to their abnormal posture for long working hours. The chief complaints were noted as neck pain presenting with forward head and neck posture. There is need for more researchers in work related musculoskeletal disorders to be studied with respect to profession, age, gender and the chief complains, psychological issues, work performance and the limitations this condition makes for an individual in his/her life24,25.

Although the prevalence and incidence of Upper Cross Syndrome (UCS) have been studied in different populations, including laundry workers to office workers, from students to various health care professionals, among the best of author's 
knowledge, this is the first study addressing the prevalence of UCS among physiotherapists community working in Pakistan, besides this study has established the strong correlation of Upper Cross Syndrome with Work Related Musculoskeletal Disorders (WRMSDs).

In recent COVID crises, the data was collected electronically through emails or social media platforms as well as screening was based upon the participant's perception that might lead towards the biasness. Furthermore, sample size was not completely fulfilled due to ongoing pandemic. Since the physiotherapy employment outlook is very competitive, it is possible that some respondents misconstrued or even masked their injury to demonstrate their competency for the job. Moreover, it is observed that individuals involved in physical activity has low prevalence of Work-Related Musculoskeletal Disorders (WRMDs) so in this study participants involved in physical activities were not ruled out.

The Upper Cross Syndrome (UCS) is an understudied phenomenon despite physiotherapists are alleged to have sufficient understanding of correct posture and adequate knowledge of musculoskeletal disorders, they're at high risk of developing Work-Related Musculoskeletal Disorders (WRMDs). To date, there are several studies reported to ascertain the frequency and profile of Work-Related Musculoskeletal Disorders (WRMDs) among Physiotherapist (PTs), however there's lack of data regarding prevalence of Upper Cross Syndrome (UCS) among Physiotherapist (PTs) in Pakistan. Therefore, future researches are required to gauge the burden of the musculoskeletal disorders to strategize effective prevention and management strategies. Moreover, there is no data available to evaluate the psychological stress, work performance and its relationship with Work Related
Musculoskeletal Disorders (WRMDs) especially in healthcare professional of Pakistan.

\section{CONCLUSION}

It was concluded that Upper Cross Syndrome (UCS) prevalence was found to be $27 \%$ in working physiotherapists whereas female and individuals working for prolonged duration were found to be more prone to develop Upper Cross Syndrome (UCS) and it is also found that there is a strong correlation with Work Related Musculoskeletal Disorders (WRMDs) with Upper Cross Syndrome (UCS). Further studies should be conducted taking large sample size and confounding factors for the better understanding of the health-related outcomes.

\section{REFERENCES}

[1] James, S.L., Abate, D., Abate, K.H., Abay, S.M., Abbafati, C., Abbasi, N., Abbastabar, H., Abd-Allah, F., Abdela, J., Abdelalim, A. and Abdollahpour, I., 2018. Global, regional, and national incidence, prevalence, and years lived with disability for 354 diseases and injuries for 195 countries and territories, 1990-2017: a systematic analysis for the Global Burden of Disease Study 2017. The Lancet, 392(10159), pp.1789-1858.

[2] Brennan-Olsen, S.L., Cook, S., Leech, M.T., Bowe, S.J., Kowal, P., Naidoo, N., Ackerman, I.N., Page, R.S., Hosking, S.M., Pasco, J.A. and Mohebbi, M., 2017. Prevalence of arthritis according to age, sex and socioeconomic status in six low- and middle-income countries: analysis of data from the World Health Organization study on global AGEing and adult health (SAGE) Wave 1. BMC musculoskeletal disorders, 18(1), p.271.

[3] Cerezo-Téllez, E., Torres-Lacomba, M., Mayoral-del Moral, O., SánchezSánchez, B., Dommerholt, J. and 
Gutiérrez-Ortega, C., 2016. Prevalence of myofascial pain syndrome in chronic non-specific neck pain: a population-based cross-sectional descriptive study. Pain Medicine, 17(12), pp.2369-2377.Chiropratic, 18(24).

[4] Bevan S. Economic impact of musculoskeletal disorders (MSDs) on work in Europe. Best Practice \& Research Clinical Rheumatology. 2015 Jun 1;29(3):356-73.

[5] Vieira, E.R., Schneider, P., Guidera, C., Gadotti, I.C. and Brunt, D., 2016. Work-related musculoskeletal disorders among physical therapists: a systematic review. Journal of back and musculoskeletal rehabilitation, 29(3), pp.417-428.

[6] Vieira, E.R., Svoboda, S., Belniak, A., Brunt, D., Rose-St Prix, C., Roberts, L. and da Costa, B.R., 2016. Workrelated musculoskeletal disorders among physical therapists: an online survey. Disability and rehabilitation, 38(6), pp.552-557

[7] Rajalaxmi, V., Paul, J., Nithya, M., Lekha, S.C. and Likitha, B., 2018. Effectiveness of three dimensional approach of schroth method and yoga on pulmonary function test and posture in upper crossed syndrome with neck Pain-A double blinded study. Research Journal of Pharmacy and Technology, 11(5), pp.1835-1839.

[8] Page, P., Frank, C.C. and Lardner, R., 2010. Assessment and treatment of muscle imbalance: the Janda approach (pp. 49-55). Champaign, IL: Human kinetics.

[9] Christensen, K., 2000. Manual muscle testing and postural imbalance. Dynamic

[10] Daneshmandi, H., Harati, J. and Fahim Poor, S., 2017. Bodybuilding links to upper crossed syndrome. Physical Activity Review, 5, pp.124-131.

[11] Janda, V., Frank, C. and Liebenson, C., 1996. Evaluation of muscular imbalance. Rehabilitation of the spine: a practitioner's manual, 6, pp.97-112.

[12] hairy, W.A., Bekhet, A.H., Sayed, B., Elmetwally, S.E., Elsayed, A.M. and Jahan, A.M., 2019. Prevalence, Profile, and Response to Work-Related Musculoskeletal Disorders among Egyptian Physiotherapists. Open access Macedonian journal of medical sciences, 7(10), p.1692.

[13] Rahimi, F., Kazemi, K., Zahednejad, S., López-López, D. and Calvo-Lobo, C., 2018. Prevalence of Work-Related Musculoskeletal Disorders in Iranian Physical Therapists: A Cross-sectional Study. Journal of manipulative and physiological therapeutics, 41(6), pp.503-507.

[14] Abaraogu, U.O., Ezema, C.I. and Nwosu, C.K., 2017. Job stress dimension and work-related musculoskeletal disorders among southeast Nigerian physiotherapists. International Journal of Occupational Safety and Ergonomics, 23(3), pp.404409.

[15] Shahid, S., Tanveer, F. and Dustgir, A., Prevalence and Risk Factors for the Development of Upper-Crossed Syndrome (UCS) among DPT Students of University of Lahore. Age, 19, pp.098.

[16] Iqbal, Z. and Alghadir, A., 2015. Prevalence of work-related musculoskeletal disorders among physical therapists. Med $\mathrm{Pr}, 666(4)$, pp.459-69.

[17] Trinkoff, A.M., Le, R., Geiger-Brown, J., Lipscomb, J. and Lang, G., 2006. Longitudinal relationship of work hours, mandatory overtime, and oncall to musculoskeletal problems in nurses. American journal of industrial medicine, 49(11), pp.964-971.

[18] Tolu, S. and Başaran, B., 2019. Workrelated musculoskeletal disorders in anesthesiologists: A cross-sectional study on prevalence and risk factors. 
[19] Plange EB. Work-Related Musculoskeletal Disorders among Physiotherapists in Ghana: Prevalence, Perceived Causes and Coping Strategies (Doctoral dissertation, University Of Ghana).

[20] Shahid, S., Effect of Forward Head Posture on Neck Disability and Level of Stress among Undergraduate Students.

[21] Anyfantis, I.D. and Biska, A., 2018. Musculoskeletal disorders among greek physiotherapists: traditional and emerging risk factors. Safety and health at work, 9(3), pp.314-318.

[22] Mubeen, I., Malik, S., Akhtar, W., Iqbal, M., Asif, M., Arshad, A., Zia, S. and Khalid, S., 2016. Prevalence of Upper Cross Syndrome among the Medical Students of University Of Lahore. International Journal of Physiotherapy, 3(3), pp.381-384.

[23] Mujawar JC, Sagar JH. Prevalence of upper cross syndrome in laundry workers. Indian Journal of Occupational and Environmental Medicine. 2019 Jan;23(1):54.

[24] Hertling, D. and Kessler, R.M., 2006. Management of common musculoskeletal disorders: physical therapy principles and methods. Lippincott Williams \& Wilkins.

[25] Kumar, B., 2016. Poor posture and its causes. Int J Phys Educ Sports Health, 3, pp.177-8. 